\title{
Hydrocephalus presenting as idiopathic aqueductal stenosis with subsequent development of obstructive tumor: report of 2 cases demonstrating the importance of serial imaging
}

\author{
Jarod L. Roland, MD, ${ }^{1}$ Richard L. Price, MD, PhD, ${ }^{1}$ Ashwin A. Kamath, MD, ${ }^{1}$ S. Hassan Akbari, MD, ${ }^{1}$ \\ Eric C. Leuthardt, MD, ${ }^{1-6}$ Brandon A. Miller, MD, PhD, ${ }^{1}$ and Matthew D. Smyth, MD1
}

Departments of ${ }^{1}$ Neurological Surgery, ${ }^{2}$ Neuroscience, ${ }^{3}$ Biomedical Engineering, and ${ }^{4}$ Mechanical Engineering and Materials Science; ${ }^{5}$ Center for Innovation in Neuroscience and Technology; and ${ }^{6}$ Brain Laser Center, Washington University School of Medicine in St. Louis, Missouri

\begin{abstract}
The authors describe 2 cases of triventricular hydrocephalus initially presenting as aqueductal stenosis that subsequently developed tumors of the pineal and tectal region. The first case resembled late-onset idiopathic aqueductal stenosis on serial imaging. Subsequent imaging revealed a new tumor in the pineal region causing mass effect on the midbrain. The second case presented in a more typical pattern of aqueductal stenosis during infancy. On delayed follow-up imaging, an enlarging tectal mass was discovered. In both cases hydrocephalus was successfully treated by cerebrospinal fluid diversion prior to tumor presentation. The differential diagnoses, diagnostic testing, and treatment course for these unusual cases are discussed. The importance of follow-up MRI in cases of idiopathic aqueductal stenosis is emphasized by these exemplar cases.
\end{abstract}

https://thejns.org/doi/abs/10.3171/2017.5.PEDS1779

KEY WORDS hydrocephalus; aqueductal stenosis; pineal region; germ cell tumor; tectal glioma

$\mathrm{P}$ INEAL region and tectal tumors often present with hydrocephalus. Tumors of markedly different histopathology often present with similar symptomology due to hydrocephalus. Noncommunicating hydrocephalus typically occurs in this scenario secondary to closure of the cerebral aqueduct from an adjacent tumor. Other causes of new-onset hydrocephalus in the absence of neoplasm include infection, such as meningitis or ventriculitis, and aqueductal stenosis.

Aqueductal stenosis is a common cause of congenital noncommunicating hydrocephalus. In young children with open cranial sutures and aqueductal stenosis, increasing head size frequently leads to diagnosis. Rarely, primary aqueductal stenosis may present late in adolescence or in adulthood. In both situations, diagnosis of primary aqueductal stenosis requires advanced imaging to rule out other etiologies such as mass lesions and clinical history to exclude postinfectious or posthemorrhagic hydrocephalus.

Here we present 2 cases involving pediatric patients presenting with cryptogenic triventricular hydrocephalus in the absence of a mass lesion or aqueductal compression. Interestingly, the cerebral aqueduct was noted to be open on high-resolution structural MRI in the first case. After successful CSF diversion by endoscopic third ventriculostomy (ETV) and ventriculoperitoneal (VP) shunting, respectively, mass lesions causing new compression of the aqueduct were discovered on subsequent follow-up imaging. In the first case, a pineal region tumor was found 20 months after initial presentation. In the second case, a slowly growing tectal region intrinsic tumor was discovered 11 years after initial presentation. In both cases, ventriculomegaly was not present at the time of tumor diagnosis, since successful CSF diversion had previously been performed. We discuss the initial workup and differential diagnosis for new-onset acute hydrocephalus in these atypical cases. Additionally, we discuss the management and importance for follow-up high-resolution MRI in this clinical population. 


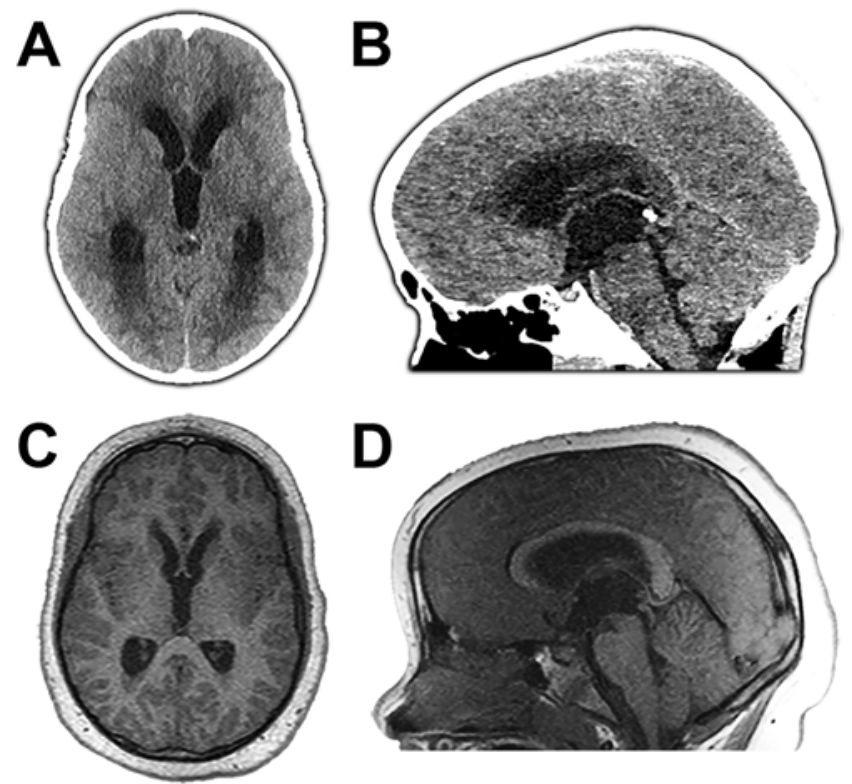

FIG. 1. Case 1. Hydrocephalus on initial presentation. A and B: Noncontrast head CT images in axial $(A)$ and sagittal $(B)$ planes obtained at initial presentation revealing triventricular ventriculomegaly consistent with noncommunicating hydrocephalus. C and D: T1-weighted MR images in axial (C) and sagittal (D) planes obtained after transfer to SLCH revealing no mass lesion and an open cerebral aqueduct.

\section{Case Reports \\ Case 1}

History and Examination

This 8-year-old boy, with a medical history significant for obesity and sinusitis, presented with an acute neurological decline over the course of 1 day. He had been in his usual state of health until the morning of presentation, when he complained of new-onset headache, nausea, and an episode of emesis.

His mental status declined and he was orotracheally intubated for airway protection at an outside hospital. Noncontrast head CT revealed triventricular hydrocephalus (Fig. 1A and B). Pineal region calcification was noted on $\mathrm{CT}$, which is not uncommon in this age group, ${ }^{25}$ and was not thought to be causal to his obstructive hydrocephalus. The child was transferred to St. Louis Children's Hospital (SLCH) for further evaluation and treatment.

On arrival to SLCH the patient was obtunded. MRI without and with contrast, including cine phase-contrast CSF flow studies, was performed (Fig. 1C and D). No enhancing abnormality was identified, the tectal region did not appear expanded, the cerebral aqueduct was patent, and no signs of tumor or mass effect were apparent. Specifically, the area of calcification noted on CT did not correlate with mass or gadolinium enhancement on MRI. MRI CSF flow studies revealed obstruction of flow at the cerebral aqueduct.

\section{Initial Operation}

We proceeded with ETV and external ventricular drain (EVD) placement. There were no complications. No ab- normalities in the ventricular system were noted during endoscopy. We obtained intraoperative CSF samples for routine infectious studies, which returned within normal limits, and Gram staining and culture revealed no growth. Postoperatively, he recovered well and was discharged to home in good condition after EVD wean. His only deficit was short-term memory difficulty.

\section{Follow-Up}

The patient returned for routine follow-up 1 week after discharge in good condition with memory improvement. Repeat MRI 4 months after presentation showed no signs of tectal or pineal region mass. His ventriculomegaly had resolved. Clinically, he had no signs or symptoms of hydrocephalus or intracranial lesion.

He was briefly lost to follow-up until 20 months postpresentation when he returned with recurrence of headaches, nausea, and a new complaint of imbalance. MRI without and with contrast revealed a new heterogeneously enhancing lesion in the pineal region, measuring approximately $2.5 \mathrm{~cm}$ in diameter, with signal characteristics of calcification and adipose tissue elements (Fig. 2A and B). We performed a lumbar puncture for CSF studies including alpha-fetoprotein (AFP), beta-human chorionic gonadotropin ( $\beta$-hCG), and carcinoembryonic antigen (CEA), which were all within normal limits. Cytological examination did not identify any signs of malignancy.

\section{Tumor Treatment}

We then performed a stereotactic needle biopsy via a right parietal transcortical approach. The biopsy pathology was consistent with mature teratoma. Due to the patient's obesity and the deep-seated tumor location, we attempted treatment with laser interstitial thermal therapy (LITT). Follow-up imaging revealed tumor progression. Therefore, we proceeded with an occipital craniotomy and transcallosal approach for gross-total resection. The patient tolerated the procedure well without complication and was discharged home on postoperative Day 4. The final pathological diagnosis from open resection was mixed germ cell tumor.

MRI performed 1 day and 3 months postoperatively confirmed gross-total resection (Fig. 2C and D). Mass effect causing compression of the aqueduct was relieved, and the aqueduct again appeared patent on structural imaging. The patient's ventricle size remained within normal limits, stable since his initial CSF diversion with ETV. Given the aggressive pathology, he was treated with carboplatin and etoposide chemotherapy. He has done well, having completed chemotherapy 7 months after the most recent surgical intervention (2.75 years after first presenting with triventricular hydrocephalus).

\section{Case 2}

\section{History and Examination}

This female infant presented at 2 months of age with findings of enlarging orbitofrontal circumference and upgaze palsy on routine clinical examination. She was born at full term via cesarean section, which was performed for failure of labor progression. The remainder of her perinatal course was unremarkable. The results of routine labo- 

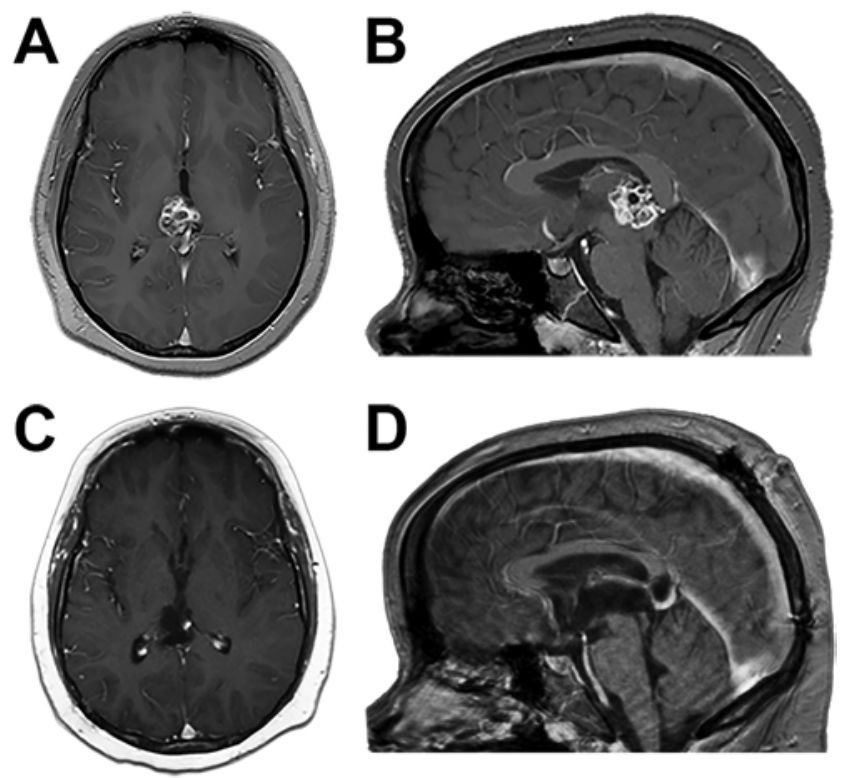

FIG. 2. Case 1. Discovery of a pineal region tumor and subsequent resection. $\mathbf{A}$ and $\mathbf{B}$ : Gd-enhanced T1-weighted axial $(A)$ and sagittal $(B)$ MR images obtained at re-presentation 20 months after development of initial symptoms, revealing a heterogeneously enhancing pineal region mass. C and D: Gd-enhanced axial (C) and sagittal (D) T1-weighted MR images obtained 3 months after open craniotomy for tumor resection showing no signs of residual tumor or recurrence. Of note, mass effect has been relieved, and the cerebral aqueduct again appears patent on postoperative structural imaging.

ratory studies were all within normal limits. The physical examination was notable for findings of a full anterior fontanel and up-gaze palsy.

Noncontrast head CT revealed triventricular ventriculomegaly consistent with obstructive hydrocephalus (Fig. $3 \mathrm{~A}$ and $\mathrm{B}$ ). High-resolution structural MRI revealed no signs of tumor or other mass lesion (Fig. 3C and D). The cerebral aqueduct appeared to taper at the rostral extent with a normal-sized fourth ventricle (Fig. 3D). Given the patient's clinical presentation and imaging findings we diagnosed aqueductal stenosis.

\section{Initial Operation}

A right occipital VP shunt was placed without complication, and postoperative CT showed reduction in ventricle size. Three months later, she returned to the clinic with vomiting. Head CT demonstrated an interval increase in ventricle size. The patient returned to the operating room for ETV and ligation of the distal VP shunt catheter. No abnormalities were noted in the ventricular system on endoscopy. She tolerated the procedure well and was discharged home in good condition. She returned on an elective basis 1 month later for VP shunt removal.

The patient returned to the clinic 7 months after shunt removal with complaints of headache and emesis. A head CT scan again demonstrated enlarged ventricles, suggesting ETV failure. A new right occipital VP shunt was placed without complication. In the following years, she had intermittent complaints of headache without nausea or vomiting, which were evaluated by $\mathrm{CT}$ and never required
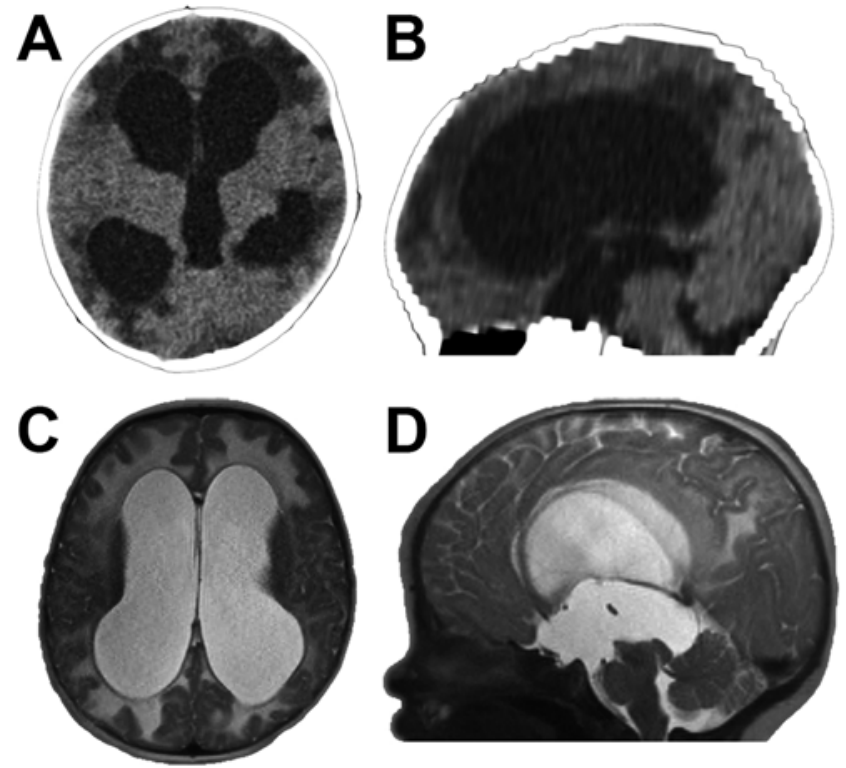

FIG. 3. Case 2. Presentation with apparent aqueductal stenosis. A and B: Axial (A) and sagittal (B) CT images obtained at initial presentation when the patient was 2 months of age revealing triventricular ventriculomegaly. C and D: Axial (C) and sagittal (D) T2-weighted MR images obtained at initial presentation demonstrating triventricular ventriculomegaly with a normal-sized fourth ventricle and tapering of the cerebral aqueduct at the rostral extent consistent with aqueductal stenosis.

shunt revision. However, when she was 11 years old, MRI was performed for evaluation of headache and revealed a tectum-based lesion expanding into the quadrigeminal cistern and compressing the cerebral aqueduct. She did not have ventriculomegaly due to CSF diversion via the VP shunt.

\section{Tumor Treatment}

We performed a supracerebellar infratentorial approach to the lesion. We did not alter her VP shunt, and she tolerated the procedure well without complication. She was discharged home on postoperative Day 4 . The final pathological diagnosis was WHO Grade I pilocytic astrocytoma.

\section{Follow-Up}

Postoperative imaging confirmed gross-total resection (Fig. 4C and D) and relief of mass effect on the aqueduct. The patient's ventricle size continues to remain small. Given the low-grade histopathology of her tumor she did not require adjuvant treatment. She continues to do well at 6 months postresection (11.5 years after initial presentation).

\section{Discussion}

Aqueductal stenosis is characterized by obstruction of CSF flow through the cerebral aqueduct in the absence of a mass lesion. The obstruction of outflow from the third to the fourth ventricle results in triventricular dilation involving the lateral and third ventricles, while sparing the fourth ventricle. Arachnoid webs, strictures, or other glial 


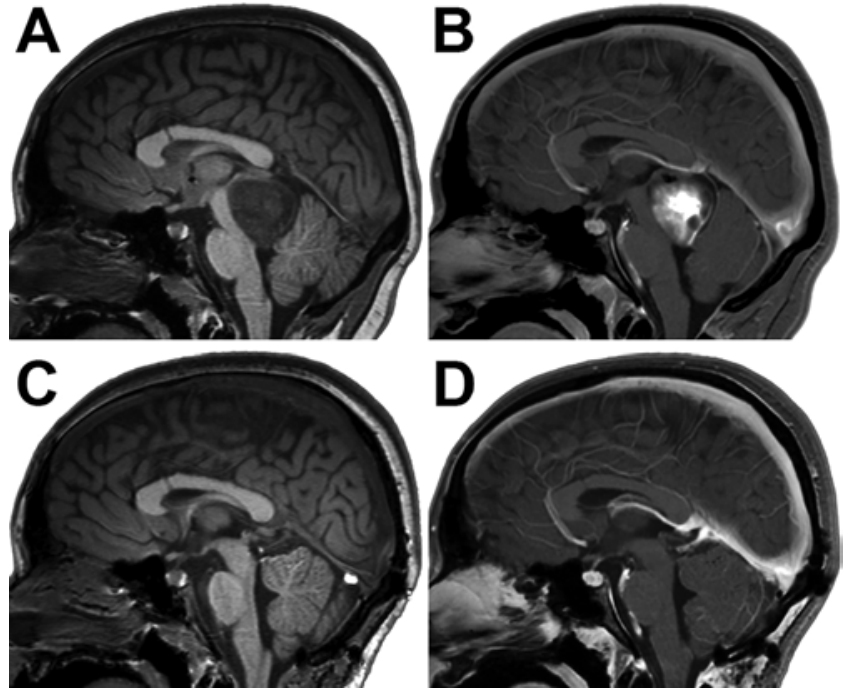

FIG. 4. Case 2. Discovery of a tectal glioma and subsequent resection. A and B: Sagittal T1-weighted MR images obtained without (A) and with $(B)$ gadolinium when the patient was 11 years old, revealing a heterogeneously enhancing tectum-based lesion causing mass effect on the cerebral aqueduct. She subsequently underwent craniotomy for tumor resection. C and D: Postoperative sagittal T1-weighted MR images obtained without $(C)$ and with (D) gadolinium revealing gross-total resection of the tumor. Of note, the patient's ventricle size remained stable throughout due to continued function of her VP shunt.

tissue within the aqueduct are common causes of obstruction and are difficult to detect on structural MRI. ${ }^{3}$ Therefore, idiopathic aqueductal stenosis may be present in the setting of visible stenosis or a structurally patent aqueduct with occult occlusion. ${ }^{22,23}$ In this setting, advanced MRI sequences utilizing cardiac-gated cine phase-contrast imaging to measure CSF flow dynamics may reveal absence of flow through the aqueduct. ${ }^{7,18,19}$ In the appropriate clinical context, MRI CSF flow studies may help identify an etiology and guide treatment decisions. ${ }^{23,26}$ Detection of tumors in the periaqueductal region usually obviates the need for CSF flow studies.

Pineal region tumors often present with a similar pattern of obstructive hydrocephalus due to mass effect on the tectal plate. Presenting symptoms are typically attributable to hydrocephalus rather than tectal compression. The signs and symptoms include headache, nausea, emesis, lethargy, gait disturbance, memory disturbance, blurred vision, and papilledema. Signs and symptoms not directly related to hydrocephalus and more specific to the pineal and tectal region may include vertical gaze palsy (Parinaud syndrome), loss of convergence, polyuria, polydipsia, and endocrinopathy. ${ }^{20}$

Tectal plate tumors may also cause obstructive hydrocephalus. These neoplasms are often slow-growing lowgrade gliomas, 2,9 and gadolinium enhancement may not be apparent, particularly early in the course. Therefore, serial MRI follow-up of tectal gliomas with attention to the tectal plate on FLAIR and gadolinium-enhanced sequences is necessary as progression to higher-grade gliomas has been described in the literature. ${ }^{17}$

The geometrical pattern of the aqueduct may provide useful information in differentiating benign idiopathic aqueductal stenosis from external compression due to mass lesion. A funnel- or trumpet-shaped aqueduct that tapers in a rostral-caudal direction, and possibly is expanded at the rostral extent, is more often associated with the benign pattern of idiopathic aqueductal stenosis. ${ }^{13,14}$

Late-onset idiopathic aqueductal stenosis (LIAS) is a rare entity but has been well described in the literature. Early descriptions of this clinical entity predate CT and therefore relied on ventriculography for diagnosis. ${ }^{11,16}$ Retrospect suggests that some such cases may have been misdiagnosed tumors due to inadequate imaging available at the time. ${ }^{17}$ More recent literature provides a modern interpretation and largely identifies ETV as an initial treatment option. ${ }^{4,5,8,10,21,24}$ The first case described in this paper initially appeared consistent with LIAS. However, a careful review of the literature provides clues to help distinguish this clinical entity.

Several case series suggest chronicity of onset to be more common in LIAS. ${ }^{6,11,12}$ Although, acute onset of neurological symptoms attributable to LIAS has previously been reported. ${ }^{15}$ Therefore, to distinguish cases of LIAS from those of congenital or tumor-related etiology, Fukuhara and Luciano proposed the following criteria: 1) no intracerebral lesions except hydrocephalus; 2) enlarged lateral and third ventricles with a comparatively small fourth ventricle; 3) no apparent history of intracerebral bleeding or meningitis; 4) no prior surgical treatment for hydrocephalus; and 5) undiagnosed in infancy. ${ }^{6}$ In a subsequent study, Locatelli et al. applied similar criteria and emphasized the clinical presentation and radiographic interpretation in the diagnosis of LIAS. Unfortunately, their only patient with an acute presentation was lost to followup at 3 months after ETV. ${ }^{12}$

In our first case, the timeline of presentation was the main finding not consistent with LIAS. Additionally, newonset obstructive hydrocephalus in adolescence is more commonly related to neoplasia than to aqueductal stenosis. Therefore, emphasis for diagnosis should be placed on clinical presentation in addition to radiographic evaluation when considering the diagnosis of adolescent-onset aqueductal stenosis.

Only 1 other report in the literature describes 2 similar pediatric patients who presented with idiopathic obstructive hydrocephalus and were only later found to have a pineal tumor on follow-up MRI. ${ }^{1}$ The authors' recommendations were for repeated MRI with contrast in all cases of new-onset hydrocephalus without a clear etiology.

\section{Conclusions}

These cases illustrate triventricular hydrocephalus without tumor on initial MRI and re-presentation in delayed fashion with a new mass lesion causing aqueductal compression. The first case appeared consistent with LIAS, with the exception of an acute time course, which is atypical. The second case presented in a more typical pattern of idiopathic aqueductal stenosis during infancy. Both patients were subsequently found to have tumors that caused occlusion of the cerebral aqueduct. Interestingly, both had successful CSF diversion that prevented devel- 
opment of symptomatic hydrocephalus. This may have delayed the tumor diagnosis until compressive symptoms were apparent.

Aqueductal stenosis is typically a benign disease that responds well to CSF diversion. However, it is prudent to perform delayed high-resolution MRI to confirm the diagnosis of idiopathic aqueductal stenosis and rule out occult slow-growing neoplasia.

\section{Acknowledgments}

Dr. Roland received support from the NINDS grant number R25NS090978-01.

\section{References}

1. Allan R, Chaseling R, Graf N, Dexter M: Aqueduct stenosis-?Benign. J Clin Neurosci 12:178-182, 2005

2. Antunes NL, Tavora L, Souweidane M: Globular glioma of the tectum. Pediatr Neurol 21:492-495, 1999

3. Arienta C, Cappricci E, Baiguini M: Non tumoral aqueduct stenosis. Multiple microscopic subependymal glial protuberances demonstrated at hystological examination. J Neurosurg Sci 30:67-70, 1986

4. Burtscher J, Bartha L, Twerdy K, Eisner W, Benke T: Effect of endoscopic third ventriculostomy on neuropsychological outcome in late onset idiopathic aqueduct stenosis: a prospective study. J Neurol Neurosurg Psychiatry 74:222-225, 2003

5. Cinalli G, Spennato P, Nastro A, Aliberti F, Trischitta V, Ruggiero C, et al: Hydrocephalus in aqueductal stenosis. Childs Nerv Syst 27:1621-1642, 2011

6. Fukuhara T, Luciano MG: Clinical features of late-onset idiopathic aqueductal stenosis. Surg Neurol 55:132-137, 2001

7. Kadowaki C, Hara M, Numoto M, Takeuchi K, Saito I: Cine magnetic resonance imaging of aqueductal stenosis. Childs Nerv Syst 11:107-111, 1995

8. Kelly PJ: Stereotactic third ventriculostomy in patients with nontumoral adolescent/adult onset aqueductal stenosis and symptomatic hydrocephalus. J Neurosurg 75:865-873, 1991

9. Kershenovich A, Silman Z, de Rungs D, Koral K, Gargan L, Weprin B: Tectal lesions in children: a long-term follow-up volumetric tumor growth analysis in surgical and nonsurgical cases. Pediatr Neurosurg 51:69-78, 2016

10. Kita D, Hayashi Y, Kitabayashi T, Kinoshita M, Okajima M, Taniguchi T, et al: Detection of the development of late-onset idiopathic aqueductal stenosis (LIAS) by chronological magnetic resonance imaging: a case report. Childs Nerv Syst 30:1317-1319, 2014

11. Little JR, Houser OW, MacCarty CS: Clinical manifestations of aqueductal stenosis in adults. J Neurosurg 43:546-552, 1975

12. Locatelli M, Draghi R, DI Cristofori A, Carrabba G, Zavanone M, Pluderi M, et al: Third ventriculostomy in late-onset idiopathic aqueductal stenosis treatment: a focus on clinical presentation and radiological diagnosis. Neurol Med Chir (Tokyo) 54:1014-1021, 2014

13. Longatti P, Fiorindi A, Perin A, Martinuzzi A: Endoscopic anatomy of the cerebral aqueduct. Neurosurgery 61 (3 Suppl):1-6, 2007

14. McCoy MR, Klausner F, Weymayr F, Georg L, Broussalis E, Golaszewski SM, et al: Aqueductal flow of cerebrospinal fluid (CSF) and anatomical configuration of the cerebral aqueduct (AC) in patients with communicating hydrocephalus - the trumpet sign. Eur J Radiol 82:664-670, 2013

15. Mucchiut M, Valentinis L, Tuniz F, Zanotti B, Skrap M, Bergonzi P, et al: Adult aqueductal stenosis presenting as a thunderclap headache: a case report. Cephalalgia 27:1171-1173, 2007

16. Nag TK, Falconer MA: Non-tumoral stenosis of the aqueduct in adults. BMJ 2:1168-1170, 1966

17. Pollack IF, Pang D, Albright AL: The long-term outcome in children with late-onset aqueductal stenosis resulting from benign intrinsic tectal tumors. J Neurosurg 80:681-688, 1994

18. Quencer RM: Intracranial CSF flow in pediatric hydrocephalus: evaluation with cine-MR imaging. AJNR Am J Neuroradiol 13:601-608, 1992

19. Quencer RM, Post MJ, Hinks RS: Cine MR in the evaluation of normal and abnormal CSF flow: intracranial and intraspinal studies. Neuroradiology 32:371-391, 1990

20. Raimondi AJ, Tomita T: Pineal tumors in childhood. Epidemiology, pathophysiology, and surgical approaches. Childs Brain 9:239-266, 1982

21. Spennato P, Tazi S, Bekaert O, Cinalli G, Decq P: Endoscopic third ventriculostomy for idiopathic aqueductal stenosis. World Neurosurg 79 (2 Suppl):S21.e13-S21.e20, 2013

22. Steel T, Maixner WJ, Chaseling R, Johnston I: Demonstration of membranous aqueduct occlusion by fast multiphase magnetic resonance imaging. J Clin Neurosci 4:352-354, 1997

23. Stoquart-El Sankari S, Lehmann P, Gondry-Jouet C, Fichten A, Godefroy O, Meyer ME, et al: Phase-contrast MR imaging support for the diagnosis of aqueductal stenosis. AJNR Am J Neuroradiol 30:209-214, 2009

24. Tisell M: How should primary aqueductal stenosis in adults be treated? A review. Acta Neurol Scand 111:145-153, 2005

25. Whitehead MT, Oh C, Raju A, Choudhri AF: Physiologic pineal region, choroid plexus, and dural calcifications in the first decade of life. AJNR Am J Neuroradiol 36:575-580, 2015

26. Yamada S, Tsuchiya K, Bradley WG, Law M, Winkler ML, Borzage MT, et al: Current and emerging MR imaging techniques for the diagnosis and management of CSF flow disorders: a review of phase-contrast and time-spatial labeling inversion pulse. AJNR Am J Neuroradiol 36:623-630, 2015

\section{Disclosures}

Dr. Leuthardt reports a consultant relationship with Monteris.

\section{Author Contributions}

Conception and design: Roland, Leuthardt, Miller, Smyth. Acquisition of data: Roland, Price. Analysis and interpretation of data: all authors. Drafting the article: Roland, Price, Miller, Smyth. Critically revising the article: all authors. Reviewed submitted version of manuscript: all authors. Approved the final version of the manuscript on behalf of all authors: Roland. Administrative/ technical/material support: Smyth. Study supervision: Smyth.

\section{Correspondence}

Jarod L. Roland, Department of Neurological Surgery, Washington University School of Medicine in St. Louis, Campus Box 8057, 660 South Euclid Ave., St. Louis, MO 63110. email: rolandj@wustl.edu. 